\section{Cardiac characterization of sgca-null mice using high resolution echocardiography}

\author{
Abdallah Fayssoil, ${ }^{1}$ Gilles Renault, ${ }^{2}$ \\ Nicolas Guerchet, ${ }^{3}$ \\ Carmen Marchiol-Fournigault, 2 \\ Françoise Fougerousse, ${ }^{3}$ \\ Isabelle Richard ${ }^{3}$
}

1Raymond Poincare Hospital, University of Versailles Saint-Quentin-en-Yvelines, Garches; 2Institut Cochin, Université Paris Descartes, Paris; ${ }^{3}$ Genethon, Evry, France

\section{Abstract}

Limb-girdle muscular dystrophy 2D (LGMD2D) is an inherited myogenic disorder belonging to the group of muscular dystrophies. Sgca-null mouse is a knock-out model of LGMD2D. Little is known about cardiac phenotype characterization in this model at different ages. We conducted a prospective study to characterize cardiac sgca-null mice phenotype using high resolution Doppler echocardiography at different ages. Conventional echocardiography was performed on anesthetised mice using a Vevo 770 (Visualsonics) with 30 $\mathrm{MHz}$ cardiac probe. Wild Type (WT) and $s g c a-$ null mice were scanned at 13,15 and 17 months. From M-mode, we measured interventricular septal (IVS) wall thickness, posterior wall (PW) thickness, and end-left ventricular diameter in systolic and diastolic. From the above parameters, we calculated left ventricular (LV) shortening fraction (SF), LV ejection fraction (EF) and LV mass. At age 13 months, PW diastolic thickness was increased in sgca-null mice $(0.89 \pm 0.14 \mathrm{~mm}$ vs $0.73 \pm 0.2$ $\mathrm{mm} ; \mathrm{P}=0.020$ ) and $\mathrm{LV}$ mass was higher in sgcanull mice (LV mass $205.2 \mathrm{mg}$ vs $143 \mathrm{mg}$; $\mathrm{P}=0.001$ ). We found also dilation of the LV (LVEDD: $4.84 \mathrm{~mm}$ vs $4.29 \mathrm{~mm} ; \mathrm{P}=0.019$ ) in sgca-null mice. At age 15 months, dilation of the LV (LVEDD: $4.86 \mathrm{~mm}$ vs $4 \mathrm{~mm} ; \mathrm{P}=0.05$ ) with an increase of the LV mass (165.7 $\mathrm{mg} v \mathrm{vs}$ 127.12; $\mathrm{P}=0.03$ ) are found in sgca-null mice. At age 17 months, we found a decrease of the PW thickening ( $17 \%$ vs $30 \% ; \mathrm{P}=0.036)$.

This work provides echocardiographic insights for the assessment of pharmaceutical therapies in sgca-null mice.

\section{Introduction}

LGMD2D is an inherited myogenic disorder belonging to the group of muscular dystrophies. LGMD2D (alpha-sarcoglycanopathy) is an autosomal recessive limb-girdle muscular dystrophy caused by mutation in the alpha sarcoglycan gene. Alpha-sarcoglycan is a component of the dystrophin glycoprotein complex (DGC) which provides stability to sarcolemma. DGC is a membrane-spanning sarcolemmal protein complex that forms a link between extracellular matrix and cytoskeletal proteins inside the cell. This link is thought to have a structural and mechanical function, and likely plays a part in other cellular activities such as signalling pathways. Alpha-sarcoglycan (SG) is a type I trans-membrane protein mainly expressed in skeletal muscle and in a lower extent in heart tissue. ${ }^{1}$ Deficiency in these proteins leads to myofiber damage following muscle contraction and consequently to muscular dystrophy. Clinically, muscular dystrophy is characterized by progressive muscle wasting and weakness of variable distribution and severity. LGMD are characterized by heterogeneous clinical presentation with weakness of pelvic and shoulder girdles and occasionally heart problem. ${ }^{2}$ Sgca-null mouse is a knockout model of LGMD2D. Little is known about heart phenotype characterization in this model at different ages. A precise characterization of the phenotype will help to perform relevant pharmacological trials and to assess gene therapy. Doppler echocardiography is a non invasive procedure that allows analysis of cardiac morphology and function. The accuracy of this procedure has been clearly demonstrated to be parallel to invasive measurements for the assessment of murine cardiac function. ${ }^{3}$ We conducted a prospective study to characterize cardiac sgca-null mice phenotype using high resolution Doppler Echocardiography (Vevo 770 , Visual Sonics) with a $30 \mathrm{MHz}$ cardiac probe.

\section{Materials and Methods}

\section{Animals}

Six C57Bl10 (WT) mice and ten sgca-null mice are included in our study. All mice were handled in accordance with the Guidelines of the Genethon committee. Each animal was shaven from the left sternal border to the left axillary line with depilatory cream. Mice were anaesthetized with Isoflurane before performing echocardiography. Initially, mouse was placed in an induction chamber with constant inflow of $3 \%$ Isoflurane mixed to oxygen. Then, mouse was removed from the induction chamber and placed on a heating platform with electrocardiogram (ECG) contact pads for ECG monitoring. Meanwhile, nose was placed into a nose cone with $1 \%$ Isoflurane in $100 \%$ oxygen. Mouse was kept on a heating pad in supine position. Ultrasound gel was applied to the
Correspondence: Abdallah Fayssoil, Raymond Poincare Hospital, APHP, University of Versailles SQY, 104 Boulevard Raymond Poincaré, 92380 Garches, France. Tel. +33.147.107.900

E-mail: fayssoil2000@yahoo.fr

Key words: Sgca-null mice, alpha-sarcoglycanopathy, LV function, echocardiography.

Contributions: the authors contributed equally.

Conflict of interests: the authors declare no potential conflict of interests.

Received for publication: 26 July 2013.

Accepted for publication: 26 August 2013.

This work is licensed under a Creative Commons Attribution NonCommercial 3.0 License (CC BYNC 3.0).

(C) Copyright A. Fayssoil et al., 2013

Licensee PAGEPress, Italy

Neurology International 2013; 5:e22

doi:10.4081/ni.2013.e22

chest of mouse before echocardiography performing.

\section{Echocardiography}

Conventional echocardiography was performed on anesthetised mice using a Vevo 770 (Visual sonics) with a $30 \mathrm{MHz}$ cardiac probe (RMV707B) .WT mice and Sgca-null mice were followed and scanned at 13,15 and 17 months. During the procedure, heart rate (HR) and temperature were monitored. For echocardiography recording, we optimized sweep speed, depth, focus and gain settings to obtain best possible images. Two-D and M-mode echocardiography were obtained from long axis view at the level of the largest LV diameter (Figure 1). $\mathrm{LV}$ dimensions (LV end-diastolic diameter = LVEDD, LV end-systolic diameter = LVESD), posterior wall (PW) and interventricular septum (IVS) wall thickness were measured using the leading edge convention of the American Society of Echocardiography (ASE). SF and EF of the left ventricle, PW thickening (PWth) and LV mass were calculated from the above dimensions (Figure 1).

\section{Statistical analysis}

Data are expressed as means \pm SD. Echocardiography parameters were compared between WT mice and sgca null mice, using Student's $t$ test. A P-value of less than 0.05 was considered statistically significant.

\section{Results}

WT mice and Sgca-null mice were similar 
regarding weight and HR at all ages except for the weight at 17 months (Tables 1-3).

At age 13 months, we found significant anatomical differences regarding PW diastolic thickness, as compared to control group $(0.89 \pm 0.14$ vs $0.73 \pm 02 ; \mathrm{P}=0.020)$. $\mathrm{LV}$ mass was significantly higher in sgca-null mice (LV mass $205.2 \mathrm{mg}$ vs $143 \mathrm{mg} ; \mathrm{P}=0.001$ ) (Table 1). Sgcanull mice at 13 months, disclosed dilation of the LV (LVEDD: $4.84 \mathrm{~mm}$ vs $4.29 \mathrm{~mm}$; $\mathrm{P}=0.019$ ). However, left ventricular systolic function (EF) was similar in the 2 groups $(0.50 \pm 6$ vs $0.47 \pm 0.02, \mathrm{P}=0.23)$.

At age 15 months, we found dilation of the LV (LVEDD: $4.86 \mathrm{~mm}$ vs $4 \mathrm{~mm}$; $\mathrm{P}=0.05$ ) in sgcanull mice with an increase of the LV mass (165.7 mg vs $127.12 ; \mathrm{P}=0.03$ ) (Table 2).

At age 17 months, we found a decrease of the PW thickening ( $17 \%$ vs $30 \% ; \mathrm{P}=0.036)$ and an increase of the ratio $\mathrm{LV}$ mass/weight (5.6 vs $3.9 ; \mathrm{P}=0.016)$. No significant differences were found regarding the other anatomic echocardiography parameters (Table 3 ).

Figures 2 and 3 summarize and illustrate the $\mathrm{LV}$ mass and the $\mathrm{LV}$ end diastolic diameter evolution of sgca-null mice with age compared to WT.

\section{Discussion}

In this study, we analysed echocardiographic parameters in a model of LGMD2D, the sgcanull mouse. In our knowledge, no data were reported in the literature about heart function in sgca-null mice, using echocardiography. LGMD2D belongs to the group of muscular dystrophies which are histologically characterized by necrotic and regenerating fibers, increase in fiber size variation, fiber splitting and centrally located nuclei. LGMD2D is an autosomal recessive limb-girdle muscular dystrophy caused by mutations in the alpha-sarcoglycan gene. Clinically, LGMD2D is characterized by great variability, ranging from severe form with onset in the first decade of life and rapid progression, resembling Duchenne muscular dystrophy (DMD), to milder form with later onset and slower progression resembling to Becker muscular dystrophy (BMD).$^{4}$ Literature is poor about cardiac function in sarcoglycanopathies.

In our study, in sgca-null mice, we found significant increase of posterior wall thickness at age 13 months with LV dilation at age 13months. Also LV mass was increased significantly in sgca-null mice at age 13 months and age 15 months. No significant difference was found regarding LV function in sgca-null mice.

In humans, heart involvement, in muscular dystrophy, is characterized initially by a dystrophic myocardium process leading to severe fibrosis and the posterior basal segment of the
Table 1. Comparison of Wild Type (WT) mice and sgca-null mice echocardiography's parameters at 13 months of age.

\begin{tabular}{lccc} 
& Sgca-null mice $(\mathbf{n}=\mathbf{1 0})$ & WT $(\mathbf{n}=\mathbf{6})$ & P value \\
Weight $(\mathrm{g})$ & $32.5(3.7)$ & $29.6(2.7)$ & 0.13 \\
HR(bpm) & $483.9(40)$ & $453.75(43)$ & 0.198 \\
\hline IVS d (mm) & $1.03(0.15)$ & $0.95(0.14)$ & 0.28 \\
IVSs (mm) & $1.4(0.2)$ & $1.22(0.18)$ & 0.13 \\
\hline PWd $(\mathrm{mm})$ & $0.89(0.14)$ & $0.73(0.2)$ & 0.020 \\
PWs (mm) & $1.09(0.17)$ & $0.80(0.28)$ & 0.019 \\
\hline PW thickness (\%) & $0.21(11)$ & $0.19(0.08)$ & 0.68 \\
LVEDD $(\mathrm{mm})$ & $4.84(0.4)$ & $4.29(0.2)$ & 0.019 \\
\hline LVESD (mm) & $3.56(0.3)$ & $3.26(0.2)$ & 0.168 \\
SF (\%) & $0.26(3.6)$ & $0.24(0.02)$ & 0.16 \\
\hline EF (\%) & $0.50(6)$ & $0.47(0.02)$ & 0.23 \\
Mass (mg) & $205.22(23)$ & $143.3(18)$ & 0.001 \\
\hline Mass/weight & $6.39(1)$ & $4.72(0.3)$ & 0.004
\end{tabular}

Data are expressed as means \pm SD. HR, heart rate; IVS d, interventricular septum diastolic; IVS s, interventricular septum systolic; LVEDD, left ventricular end diastolic diameter; LVEDS, left ventricular end diastolic diameter; SF, shortening fraction; EF, ejection fraction.

Table 2. Comparison of WT mice and sgca-null mice echocardiographic's parameters at 15 months of age.

\begin{tabular}{lccc} 
& Sgca-null mice $(\mathbf{n}=\mathbf{1 0})$ & WT $(\mathbf{n}=\mathbf{6})$ & P value \\
Weight $(\mathrm{g})$ & $32(0.35)$ & $33(0.7)$ & 0.49 \\
HR $(\mathrm{bpm})$ & $509.05(39)$ & $451.5(32)$ & 0.027 \\
\hline IVS d (mm) & $0.79(0.11)$ & $0.80(0.06)$ & 0.92 \\
IVSs (mm) & $1.18(0.14)$ & $1.06(0.07)$ & 0.11 \\
\hline PWd (mm) & $0.82(0.14)$ & $0.78(0.08)$ & 0.51 \\
PWs (mm) & $1.04(0.2)$ & $1.07(0.04)$ & 0.73 \\
\hline PW thickness (\%) & $32.7(0.14)$ & $39(0.14)$ & 0.37 \\
LVEDD (mm) & $4.86(0.6)$ & $4.27(0.3)$ & 0.05 \\
\hline LVESD (mm) & $3.51(0.6)$ & $3.16(0.2)$ & 0.22 \\
SF (\%) & $28.2(0.05)$ & $25.8(0.03)$ & 0.33 \\
\hline EF (\%) & $54(0.06)$ & $51(0.05)$ & 0.44 \\
Mass (mg) & $165.7(36)$ & $127(16)$ & 0.037 \\
\hline Mass/weight & $5.27(1.4)$ & $3.86(0.5)$ & 0.047
\end{tabular}

Data are expressed as means \pm SD. HR, heart rate; IVS d, interventricular septum diastolic; IVS s, interventricular septum systolic; LVEDD, left ventricular end diastolic diameter; LVEDS, left ventricular end diastolic diameter; SF, shortening fraction; EF, ejection fraction.

Table 3. Comparison of WT mice and sgca-null mice echocardiographic's parameters at 17 months of age.

\begin{tabular}{lccc} 
& Sgca-null mice $(\mathbf{n}=\mathbf{1 0})$ & WT $(\mathbf{n}=\mathbf{6})$ & P value \\
Weight $(\mathrm{g})$ & $29(4.9)$ & $34.16(2.9)$ & 0.046 \\
HR $(\mathrm{bpm})$ & $524.6(36)$ & $531(34)$ & 0.74 \\
\hline IVS d (mm) & $0.86(0.9)$ & $0.84(0.07)$ & 0.83 \\
IVSs $(\mathrm{mm})$ & $1.16(1)$ & $1.11(0.12)$ & 0.73 \\
\hline PWd $(\mathrm{mm})$ & $0.96(0.2)$ & $0.814(0.07)$ & 0.15 \\
PWs $(\mathrm{mm})$ & $1.12(0.2)$ & $1.08(0.5)$ & 0.73 \\
\hline PW thickness $(\%)$ & $17(10)$ & $30(0.12)$ & 0.036 \\
LVEDD (mm) & $4.36(0.4)$ & $4.21(0.24)$ & 0.44 \\
\hline LVESD (mm) & $3.16(0.5)$ & $3.10(0.3)$ & 0.73 \\
SF (\%) & $27.8(0.06)$ & $26.5(0.05)$ & 0.70 \\
\hline EF (\%) & $53.7(0.1)$ & $51.8(0.09)$ & 0.73 \\
Mass (mg) & $161.29(36)$ & $161.29(20)$ & 0.27 \\
\hline Mass/weight & $5.6(1.3)$ & $3.9(0.6)$ & 0.016
\end{tabular}

Data are expressed as means \pm SD. HR, heart rate; IVS d, interventricular septum diastolic; IVS s, interventricular septum systolic; LVEDD, left ventricular end diastolic diameter; LVEDS, left ventricular end diastolic diameter; SF, shortening fraction; EF, ejection fraction. 
$\mathrm{LV}$ is the first site affected in muscular dystrophy. As sarcoglycan genes encode for proteins that form a supra-molecular link between the cytoskeleton and the extracellular matrix, absence of one of the SG may lead to degeneration of muscle fibre due to mechanical stress associated to contraction. Death of the muscle fibre is followed by an inflammatory reaction that results in a reactive sclerosis and reduced local microvasculature.

Sgca null mice disclose a clinical phenotype very similar to human conditions with a severe pattern of regeneration/degeneration cycles regions of mononucleated-cell infiltrate, fiber splitting and fatty infiltrations. Beside this altered histological phenotype, mice exhibit muscle specific force decrease. 5 with numerous centronucleated fibers, wide
It has been shown that deficiency of alphasarcoglycan in mice is less associated with cardiomyopathy. ${ }^{6}$ However, it would be interesting to characterize cardiac function using speckle abnormalities in mice without normal LV ejection fraction. ${ }^{7}$ Speckle tracking echocardiography (STE) is a recent technology that can assess myocardial strain using analysis of speckle motion present in a classical 2D echocardiographic image.

\section{Study limitations}

Anaesthesia agents may be deleterious on heart function in mice models. Isoflurane is known to decrease contractility and heart rate in mice. ${ }^{8}$ Heart rate values are lower in our study compared to previous studies and left tracking imaging that can detect cardiac

ventricular EF is lower in our study because of possible isoflurane effect on LV contractility. 9,10

\section{Conclusions}

The evolution over time of the LV dimensions and mass in these animal's models of muscular dystrophy are similar to early signs observed in humans. This work provides echocardiographic insights for the evaluation of the efficiency of pharmaceutical or gene cardiac therapies in sgca-null mice.

\section{References}

1. Roberds SL, Leturcq F, Allamand V, et al. Missense mutations in the adhalin gene linked to autosomal recessive muscular dystrophy. Cell 1994;78:625-33.

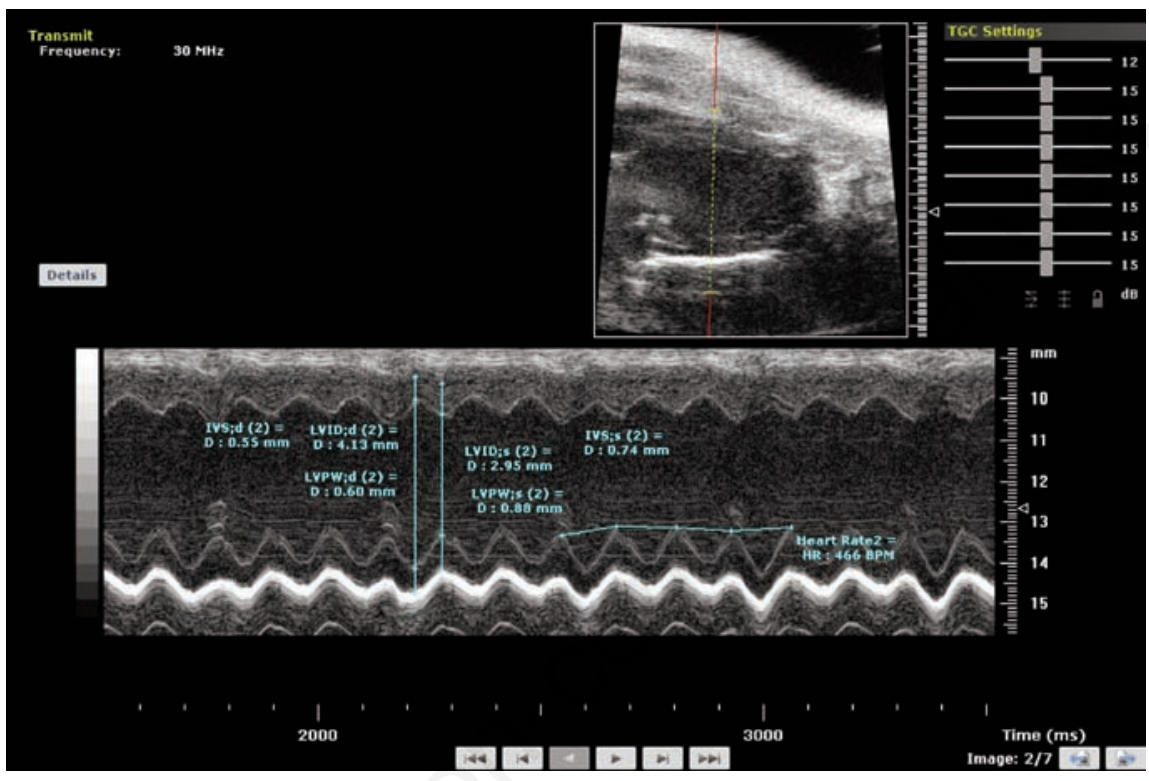

Figure 1. M-mode tracing of the left ventricle. Measurements of the LV dimensions are used for calculation of LV shortening fraction, LV ejection fraction and LV mass.
2. Finsterer J, Stöllberger C. The heart in human dystrophinopathies. Cardiology 2003;99:1-19.

3. Esposito G, Santana LF, Dilly K, et al. Cellular and functional defects in a mouse model of heart failure. Am J Physiol 2000;279:H3101-12.

4. Eymard B, Romero NB, Leturcq F, et al. Primary adhalinopathy (alpha-sarcoglycanopathy): clinical, pathologic, and genetic correlation in 20 patients with autosomal recessive muscular dystrophy. Neurology 1997;48:1227-34

5. Consolino CM, Duclos F, Lee J, et al. Muscles of mice deficient in alpha-sarcoglycan maintain large masses and near control force values throughout the life span. Physiol Genomics 2005;22:244-56.

6. Zhu X, Wheeler MT, Hadhazy M, et al. Cardiomyopathy is independent of skeletal muscle disease in muscular dystrophy. FASEB J 2002;16:1096-8.
Lv mass

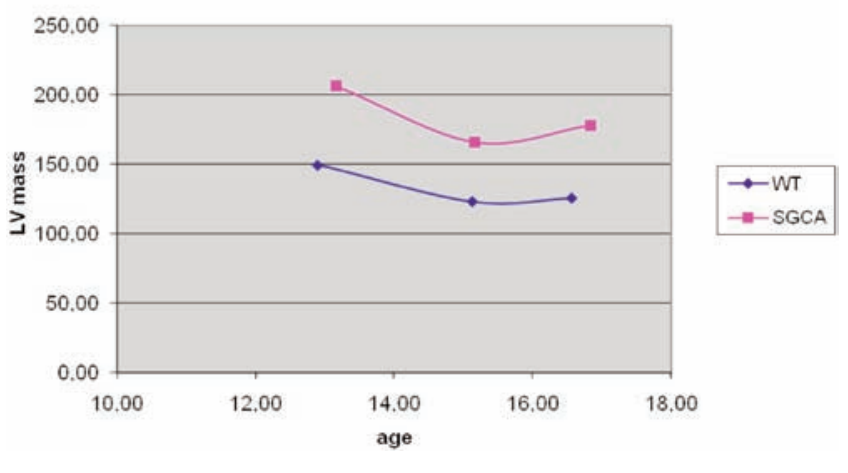

Figure 2. Evolution of the LV mass (mg) from age 13 months to 17 months in sgca-null mice and WT mice. LV mass (unit) = mg, Age $=$ months .

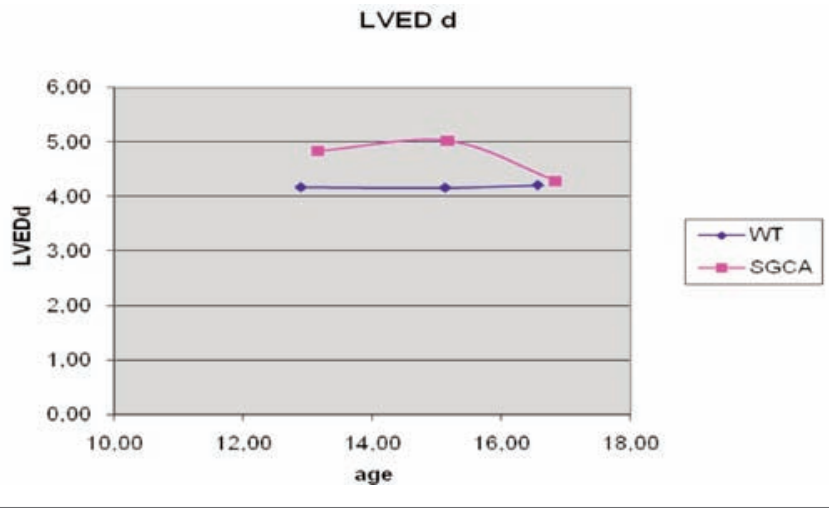

Figure 3. Evolution of the LV end-diastolic diameter (LVED d) from age 13 months to 17 months in sgca-null mice and WT mice. LVED d (unit) $=$ mm, Age = months . 
7. Peng Y, Popovic ZB, Sopko N, et al. Speckle tracking echocardiography in the assessment of mouse models of cardiac dysfunction. Am J Physiol Heart Circ Physiol 2009;297:H811-20.

8. Roth DM, Swaney JS, Dalton ND, et al. Impact of anesthesia on cardiac function during echocardiography in mice. Am $\mathrm{J}$ Physiol Heart Circ Physiol 2002;282:H2134-40.

9. Spurney CF, Knoblach S, Pistilli EE, et al. Dystrophin-deficient cardiomyopathy in mouse: Expression of Nox4 and Lox are associated with fibrosis and altered func- tional parameters in the heart. Neuromuscul Disord 2008;18:371-81.

10. Muntoni F. Cardiac complications of childhood myopathies. J Child Neurol 2003;18: 191-202. 